\title{
miscellany
}

\section{UK Psychiatrists Huntington's Disease Group}

The UK Psychiatrists Huntington's Disease Group was formed in 1999 and meets twice yearly at the Royal College of Psychiatrists. The Group consists of Members and Fellows of the College who have an interest in Huntington's disease. The aim of the Group is to improve the clinical management of Huntington's disease in the UK and in particular the management of its psychiatric consequences. The specific aims are: the development of assessment and treatment guidelines; the creation of training opportunities; the creation of a support network for psychiatrists with an interest in the disease; and the facilitation of research on the management of the disease. A website is under construction and may be found at www.huntingtons. org.uk. For further information, or to take part in the group, please write to Dr Ken Barrett, The Neurobehavioural Unit, The Haywood Hospital, High Lane, Burslem, Stoke-on-Trent, Staffordshire ST6 7AG.

\section{National launch of SANE's helpline for mental health professionals}

Following the initial success of a pilot programme in East London and Wiltshire,
SANE is expanding its dedicated professional telephone helpline nationally. Professionals will have access to a national database including information on: statutory, voluntary and independent mental health services; illnesses and symptoms; medication, treatments and side-effects: psychological treatments and other therapies; mental health law; prisons and mental health; and the Disability Discrimination Act. SANE's Professional Line is staffed by trained professionals, who will also provide access to experts throughout the UK prepared to discuss cases with fellow professionals. Many are specialists in particular fields. SANE's professional line is open from 9.00 a.m. to 9.00 p.m. Monday to Friday, and 12 noon to 9.00 p.m. at weekends: tel. : 09065585858 (calls are charged at the premium rate, currently $\mathrm{f} 1.00$ per minute).

\section{New courses from CNA's Training Unit}

The new programme for the Training Unit of Carers National Association (CNA) is now available, with details of all courses taking place from October 2000 to March 2001. The new courses described in the programme are 'Carers and their rights, under the Carers (R\&S) Act 1995, and Carers and Disabled Children Act 2000', 'The Human Rights Act 1998', 'Back to work: making it real for carers' and 'Support and accommodation for asylum seekers'. The training days are held at CNA's head offices in London and Manchester. For a free copy of CNA's Training Unit programme, or details about the cost of courses, please contact Karin Beeler, Training Unit Administrator, Carers National Association, 20-25 Glasshouse Yard, London EC1A 4JT. (tel. : 0207566 7632)

\section{New CGSS website directory}

There is now a vast amount of informa tion available on the internet that is relevant to mental health services. With so many different organisations, websites and internet browsers, however, finding it can be very time consuming and often a matter of luck. To assist clinicians, researchers, students, clinical audit and clinical governance staff in finding information without wasting valuable time, the Clinical Governance Support Service (CGSS) has produced a Website Directory for Mental Health. Comprising over 350 websites, this book comes with MS Word on disk to make accessing the sites even easier. The directory costs $\mathrm{f} 20$ and is available from the CGSS office, tel.: 0207227 0829; fax: 0207227 0850; e-mail: kirsty. maclean_steel@virgin.net.

\section{forthcoming events}

Three conferences have been organised by the University of Southampton's Mental Health Group Professional Education and Training Unit: Section 12(2) Mental Health Act 1983 refresher day, to be held on 1 November 2000, The treatment of sex offenders: is it a mental health issue? (10 November 2000) and Mentally disordered offenders in the community (22 March 2001). Further information: David Beck, the PET Unit, Mental Health Group, University of Southampton, Royal South Hants Hospital, Southampton SO14 OYG (tel./fax: 0238082 5543; e-mail: dkb@soton.ac.uk).

The Association for Psychoanalytic Psychotherapy in the NHS is holding a conference entitled The acute ward in a changing environment - therapeutic or counter-therapeutic? on 17 November at the Botanical Gardens, Birmingham. Further information: Annabel Thomas, APP, PO Box 707, Gerrards Cross, Buckinghamshire SL9 0XS (tel./fax: 01494 581 539).

Mole Conferences would like to announce the following conferences, to be held in
London: An introduction to cognitive behavioural skills (5 October 2000), Asperger's across the life-span (18 October 2000), Friends and enemies: our need to love and hate (9 November 2000), Domestic violence: working with the abused and the abusers (20 November 2000), The annual conference on religion and psychiatry: medication/culture/ control (23 November 2000), Personality disorders: mad, bad or just misunderstood (15 March 2001). Further information: Mole Conferences, 26 Church Road, Portslade, Brighton BN41 1LA (tel. : 01273242 634; fax: 01273 235 095; e-mail: enquiries@mole. conferences.com).

The 13th international psychiatric conference of Pakistan Psychiatric Society will take place in the twin cities of RawaliniIslamabad from 25 to 28 January 2001. Further information: http:www. telmedpak.com/doctors/conferences. html. The closing date for abstract submission is 31 October 2000 .

The 13th World Congress of the World Association for Dynamic Psychiatry, entitled The relevance of groups for illness, health and healing, organised in association with the German Academy for Psychoanalysis will take place on 13-17 March 2001. Further information: Ilse Burbiel, WADP (tel.: +49 895369 74; fax: +49 89532883 7; e-mail: Ifi-muenchen@dynpsych.de).

The World Federation for Mental Health will be holding its Biennial Congress in 2001 in collaboration with the Canadian Mental Health Association. The theme is Respecting diversity in mental health. Further information: The World Assembly for Mental Health 2001, c/o Venue West Conference Services, 645-375 Water Street, Vancouver, BC V6B 5C6, Canada (tel.: +1 604681 5226; fax: +1 604681 2503; e-mail: wamh2001@venuewest. com).

The Clinical Governance Support Service is organising a one-day multi-professional conference Clinical governance in forensic mental health services on 7 December 2000 in London. For a programme and booking form, contact: Michelle Procopi (tel.: 0207227 0829; e-mail: m.procopi@virgin.net). 